\title{
Short term protective effect of digitoxin in sepsis-induced acute lung injury
}

\author{
Ejder Saylav BORA ${ }^{1, *}$; Mümin Alper ERDOĞAN²; Bahattin ÖZKUL ${ }^{3}$; İbrahim Halil SEVER ${ }^{4}$; İBrahim SÖĞÜT5; \\ CANAN HÜRDAĞ $\breve{G}^{6}$; OYTUN ERBAŞ \\ ${ }^{1}$ İzmir Atatürk Research and Training Hospital Emergency Medicine, Izmir, Turkey \\ 2 Department of Physiology, İzmir Katip Çelebi University, Izmir, Turkey \\ ${ }^{3}$ Department of Radiology, Istanbul Atlas University, Istanbul, Turkey \\ ${ }^{4}$ Department of Radiology, Demiroğlu Bilim University, Istanbul, Turkey \\ ${ }^{5}$ Department of Biochemistry, Demiroğlu Bilim University, Istanbul, Turkey \\ ${ }^{6}$ Department of Histology and Embryology, Demiroğlu Bilim University, Istanbul, Turkey \\ ${ }^{7}$ Department of Physiology, Demiroğlu Bilim University, Istanbul, Turkey
}

Key words: Digitoxin, Animal model, Sepsis, Acute lung injury

\begin{abstract}
Purpose: Digitoxin is a cardiac glycoside used in the treatment of heart failure. Inspired by its known antiinflammatory effect, this study aims to investigate the effect of digoxin in a sepsis model and to bring to light its effect and underlying mechanism in acute lung injury (ALI). Method: 28 wistar albino rats were divided into 4 groups. Sepsis model is performed by the feces intraperitoneal-injection procedure (FIP). Results: TNF-a, CRP, IL-6, IL 1-Beta, lactic acid, and MDA values were significantly decreased in the FIP+digitoxin group compared to the FIP+Saline group. When the same groups were examined, histological improvements such as decrease in alveolar inflammation and decrease in septal thickening in the digitoxin group and thorax CT were found to be significantly higher in the Hounsfield unit digitoxin group compared to the Saline group. Conclusion: Digitoxin has shown biochemical improvement in sepsis with all known mechanisms of action, and healing effects in both computerized tomography and histology in the lung.
\end{abstract}

$\begin{array}{ll}\text { Abbreviations } \\ \text { ALI: } & \text { Acute Lung Injury } \\ \text { NF-kB: } & \text { Nuclear factor kappa-light-chain-enhancer of } \\ & \text { activated B } \\ \text { TNF-a: } & \text { Tumor necrosis factor-alpha } \\ \text { IL: } & \text { Interleukin } \\ \text { Th: } & \text { T helper } \\ \text { FIP: } & \text { Feces-induced peritonitis } \\ \text { I.P.: } & \text { Intraperitoneally } \\ \text { CRP: } & \text { C reactive protein } \\ \text { MDA: } & \text { Malondialdehyde } \\ \text { TBARS: } & \text { Thiobarbituric acid-reacting substances } \\ \text { PBS: } & \text { Phosphate-buffered saline } \\ \text { AC: } & \text { alveolar congestion } \\ \text { H: } & \text { hemorrhage }\end{array}$

*Address correspondence to: Ejder Saylav Bora, saylavbora@hotmail.com Received: 31 July 2021; Accepted: 08 September 2021

$\begin{array}{ll}\text { AL: } & \begin{array}{l}\text { Leukocyte infiltration or aggregation in air } \\ \text { spaces/vessel walls }\end{array} \\ \text { PE: } & \text { Perivascular/interstitial edema } \\ \text { ROIs: } & \text { Regions of interest } \\ \text { H\&E: } & \text { hematoxylin and eosin } \\ \text { SEM: } & \text { Standard error of the mean } \\ \text { SPSS: } & \text { Statistical Package for the Social Sciences } \\ \text { MCP-1: } & \text { Monocyte chemoattractant protein-1 } \\ \text { VCAM-1: } & \text { Vascular cell adhesion protein 1 } \\ \text { HU: } & \text { Hounsfield unit } \\ \text { CT: } & \text { Computerized Tomography }\end{array}$

\section{Introduction}

Sepsis and septic shock are common health problems with high mortality rates, affecting millions of patients every year. Overreaction of inflammatory mediators against infectious pathogens plays a key role in the disease development. This extreme reaction in septic shock drives a vicious circle of 
continuous damage to the capillary endothelium and tissue, and changes the blood flow in the microcirculation, the state of vascular perfusion, and organ function (Nguyen et al., 2006).

Acute lung injury (ALI) is a devastating complication of septic shock. Sepsis and ALI have similar mechanisms such as inflammation and endothelial dysfunction, and increased permeability of alveolar epithelial cells (Filgueiras et al., 2012). In addition, septic shock is the most common etiology of ALI, and patients with sepsis-triggered ALI have higher mortality rates than those with other risk factors (Nguyen et al., 2006). Early targeted therapy in patients with septic shock has reduced the proportion of patients needing mechanical ventilators (Paul, 2018). These findings suggest that the treatment of underlying sepsis and the identification of patients at risk of developing ALI are of paramount importance.

A cytokine storm is the main condition to be prevented in sepsis. A limited number of drugs can inhibit cytokine expression activated by nuclear factor kappa-light-chainenhancer of activated B cells (NF-kB) (Yang et al., 2013; Teijaro et al., 2014). Nephrotoxic and neurotoxic side effects can occur with the chronic or acute use of these drugs (Teijaro et al., 2014). An alternative that does not cause such toxicities is digitoxin: one of the most potent inhibitors of the proinflammatory tumor necrosis factor-alpha (TNF-a)/NF-kB pathway, which can prevent cytokine storms (Bette et al., 2020).

Digitoxin is a cardiac glycoside that has been used to treat heart failure for many years (Hashim et al., 2014). Although clinical studies have provided evidence of the beneficial effects of digitoxin on cardiovascular events (Hashim et al., 2014; Dec, 2003), no data on the early influence of digitoxin on sepsis are available so far. Digitoxin exerts its antiinflammatory effect by inhibiting the pro-inflammatory cytokines TNF-a, interleukin-1b (IL-1b), IL-6, and IL-8 through NF-kB blockade (Jagielska et al., 2009). It is one of the first known drugs to have anti-inflammatory effects in cystic fibrosis through the inhibition of the secretory cytokine IL-8 (Bette et al., 2020). It might also have beneficial effects on autoimmune diseases through T-helper 17 (Th-17) cell blockade (Letícia et al., 2018). It was found to be successful experimentally in uveitis, but its use has been discontinued because it causes retinal degeneration (Jagielska et al., 2009). Although its anti-inflammatory effect does not take place through this mechanism, digitoxin also reduces the body's energy needs by inhibiting the $\mathrm{Na}^{+} / \mathrm{K}^{+}$-ATPase pump.

Currently, sepsis patients are most commonly initially treated by emergency services. The first and most important step for early diagnosis and treatment is interventions in the emergency department. The hypothesis of this study is that digitoxin may ameliorate ALI by suppressing the excessive inflammatory response of sepsis. In this study, we evaluated the early protective and reversible effects on ALI of digitoxin administration in an experimental sepsis model using histology, biochemistry, and imaging methods, with the aim of presenting a new perspective to clinicians.

\section{Materials and Methods}

\section{Animals}

Thirty-four male Wistar albino rats weighing 200-250 g were used. Experimental animals were fed ad libitum and housed in steel cages with an average temperature of $22 \pm 2^{\circ} \mathrm{C}$ and 12 -h day and night cycles that were adjusted automatically.

\section{Experimental procedures}

We used a feces-induced peritonitis (FIP) rat model for sepsis, established according to the method previously described by Shrum et al. (2014) and Tyml et al. (2017). Two adult rats were sacrificed by high-dose anesthesia and cervical dislocation. Semi-moist fecal material was freshly prepared from the cecum and suspended in saline at a concentration of $75 \mathrm{mg} / \mathrm{ml}$. The solution was administered intraperitoneally (i.p.) using a 21-G injector via a single procedure at a dosage of $1 \mathrm{~g} / \mathrm{kg}$ bodyweight. The rats were randomly assigned into four groups. FIP was performed on 28 rats to induce the sepsis model. Eight rats were assigned to a normal control group and did not undergo any procedures. Four rats died during the first $24 \mathrm{~h}$ following the procedure and were excluded from the study. The four study groups ( $\mathrm{n}=8$ for each) were designed as follows: a control group that was non-operative and was orally fed; a group that underwent FIP alone; a placebo group that underwent FIP and then received i.p. $10 \mathrm{ml} / \mathrm{kg} 0.9 \% \mathrm{NaCl}$ saline; and a treatment group that underwent FIP and then received i.p. $80 \mu \mathrm{g} / \mathrm{kg} /$ day digitoxin (Digitoxin-Sandoz Ampul, $0.5 \mathrm{mg} / 2 \mathrm{~mL}$; Novartis). All treatments were administered $1 \mathrm{~h}$ after the FIP procedure. At $20 \mathrm{~h}$, computed tomography (CT) was performed on all animals under ketamine anesthesia. The study was finished after 24 $\mathrm{h}$ and euthanasia was performed using a high dose anesthesia combination of $100 \mathrm{mg} / \mathrm{kg}$ ketamine (Ketasol $10 \%$; Richterpharma) and $50 \mathrm{mg} / \mathrm{kg}$ xylazine (Rompun $2 \%$; Bayer) and cervical dislocation. Blood samples were collected before euthanasia under anesthesia by cardiac puncture for biochemical analysis.

Determination of TNF-a, C-reactive protein (CRP), $I L-6, I L-1 b$, and lactic-acid levels in plasma

Enzyme-linked immunosorbent assay (ELISA) kits (Biosciences, Abcam) were purchased and TNF-a, CRP, IL$1 \mathrm{~b}$, and IL-6 levels were measured in the blood. ELISA instructions were followed. CRP and TNF-a were determined in two replicates by diluting the plasma samples 1:2 as per the instructions. Lactic-acid levels were determined with a blood gas device.

\section{Measurement of lipid peroxidation}

Lipid peroxidation was found by measuring malondialdehyde (MDA) levels from plasma samples using thiobarbituric acid reagents. This process was carried out by mixing trichloroacetic acid and thiobarbituric acid-reacting substances (TBARS) with plasma, after incubation at $100^{\circ} \mathrm{C}$ for $60 \mathrm{~min}$, cooling on ice, centrifuging at 3,000 rpm, and reading the last supernatant at $535 \mathrm{~nm}$.

\section{Histopathological examination of lungs}

For histological study, all animals were given $100 \mathrm{mg} / \mathrm{kg}$ ketamine (Ketasol 10\%; Richterpharma) and $50 \mathrm{mg} / \mathrm{kg}$ xylazine (Rompun 2\%; Bayer) i.p., and perfused with $200 \mathrm{ml}$ of $4 \%$ formaldehyde in $0.1 \mathrm{M}$ phosphate-buffered saline (PBS). Formalin-fixed lung sections $(5 \mu \mathrm{m})$ were stained 
with hematoxylin and eosin (H\&E). All sections were photographed with an Olympus C-5050 digital camera connected to an Olympus BX51 microscope. The main histopathological lung-injury score was calculated as previously described (Kwon et al., 2011). Briefly, histopathological lung injury was assessed by scoring alveolar congestion (AC), hemorrhage $(\mathrm{H})$, leukocyte infiltration or aggregation in air spaces/vessel walls (AL), perivascular/interstitial edema (PE), and alveolar wall thickness/hyaline membrane formation (TA). The severity of each lung section was graded as 1 (0-25\%), 2 (25-50\%), 3 (50-75\%), or 4 (75-100\%) (Kwon et al., 2011).

\section{CT examination of lungs}

All examinations were performed using a 16-slice multidetector row CT scanner (Somatom Go Now, Siemens Healthcare, Erlangen, Germany) in the supine position without using contrast media after injection of the anesthetic agent. Rats were deeply anesthetized by a mixture of ketamine (80 mg/kg; Ketasol, Richterpharma AG Austria) and xylazine (10 mg/kg; Rompun, Bayer, Germany) i.p. All animals were bound on the scanning table using suitable materials to prevent motion artifacts. The scanning parameters were $120 \mathrm{kV}$ variable mAs according to the automatic exposure control system and 1-mm slice thickness. The scanning range was the C3 vertebrae to the diaphragm including the apex and base of the lung. After acquisition, all images were reconstructed at $1-\mathrm{mm}$ non-overlapping slices with a $512 \times 512$ matrix size and a sharp reconstruction kernel (KernelBr64). All images were evaluated by three radiologists who were blinded to the animals' laboratory findings and groupings. Six regions of interest (ROIs) with an equal size of $2,153 \mathrm{~mm}^{2}$ (two in the upper zone, two in the middle zone, and two in the lower zone of both lungs) were plotted on axial images with a parenchymal window at the level of near the heart apex for all animals. Care was taken to avoid large vessels, airways, and bones when plotting the ROIs. The Hounsfield Unit (HU) value was calculated in the ROIs. The HU value is 1,000 in bone (complete absorption), $-1,000$ in air (no absorption), and 0 in water. Hence, for example, a 1,000 HU voxel consists of gas alone, a $0 \mathrm{HU}$ voxel consists of water (or 'tissue') alone, and a 500 voxel HU consists of approximately $50 \%$ gas and $50 \%$ water (or tissue). In our study, the HU value was calculated from ROIs. The lung parenchyma window was based on -500 to $-600 \mathrm{HU}$ (Maurizio et al., 2014).

\section{Statistical analysis}

Data are presented as the mean \pm the standard error of the mean (SEM). Analyses were performed using SPSS version 15.0 for Windows. All analyses were done by the nonparametric (Mann-Whitney U) test. $p$ values $\leq 0.05$ were considered statistically significant.

\section{Results}

\section{Biochemical findings}

The TNF-a, CRP, IL-1b, IL-6, and MDA plasma levels were significantly higher in the FIP+saline group compared to the control group $(p<0.001)$. By contrast, the TNF-a, CRP,
IL-1b, IL-6, and MDA plasma levels in the FIP+digitoxin group were lower than the FIP+saline group $(p<0.001)$. The lactic-acid levels were significantly higher in the FIP + saline group compared to the control group $(p<0.05)$. Moreover, the lactic-acid levels in the FIP+digitoxin group were lower than the FIP+saline group $(p<0.01)$ (Tab. 1$)$. The MDA and CRP levels were significantly higher in the FIP+saline group compared to the control group $(p<$ $0.001)$, and lower in the FIP+digitoxin group than in the FIP+saline group $(p<0.01)$ (Tab. 1).

\section{Histological score and CT findings}

The AC, H, AL, PE, and TA lung damage scores were significantly higher in the FIP+saline group than the control group $(p<0.001)$. All scores in the FIP+digitoxin treatment group were significantly lower compared to the FIP+saline group (AC, AL, and PE $p<0.05 ; \mathrm{H}$ and TA $p<0.001$ ) (Fig. 1) (Tab. 2).

Histopathological lung damage was assessed by scoring alveolar congestion (AC), hemorrhage $(\mathbf{H})$, infiltration or aggregation of leukocytes in air spaces/vessel walls (AL), perivascular/interstitial edema (PE), and thickness of the alveolar wall/hyARDSne membrane formation (TA). The severity for each item was graded as 1 (\%0-25), 2 (\%25-50), 3 (\%50-75), 4 (\%75-100). ${ }^{\star} p<0.01,{ }^{* *} p<0.001$ different from normal groups; ${ }^{\#} p<0.05,{ }^{\# \#} p<0.001$ different from FIP and saline group.

The HU value of the lung in the FIP+saline group was higher than in the control group and this difference was statistically significant $(p<0.001)$. The HU value of the lung in the FIP+digitoxin treatment group was lower in the FIP + saline placebo group, and this difference was statistically significant $(p<0.001)$ (Figs. 1 and 2$)$.

\section{Discussion}

The importance of early antibiotic therapy in sepsis and septic shock to prevent ALI and limit the infectious focus has previously been established (Vincent et al., 2014). However, so far, no drug has been shown to suppress the inflammatory response and protect hemodynamics during the early stage. Our current study demonstrated evidence of the benefits of early digitoxin use in ALI.

TNF-a, IL-1b, and IL-6, which are proinflammatory cytokines, are among the first parameters to increase in the inflammatory response via NF-kB. Digitoxin has been shown to inhibit the expression of monocyte chemoattractant protein-1 (MCP-1) and vascular cell adhesion protein 1 (VCAM-1), which is an important proinflammatory mediator induced by IL- $1 \beta$, thus preventing monocytes from adhering to endothelial cells (Fürst et al., 2017). IL-6 has become an important marker in measuring the severity of sepsis and during the follow-up (Remick et al., 2005). TNF-a triggers apoptotic cell death, cachexia, and inflammation ${ }^{18}$. After the FIP procedure in our study, the TNF-a, CRP, IL-1b, IL-6, and MDA levels in the FIP alone and FIP+saline groups increased significantly, and they were inhibited by digitoxin as their values were observed to decrease significantly after treatment. Results supporting these findings were reported by Leite et al. (2018) in a Zymosan-induced peritonitis model. They showed 
TABLE 1

Biochemical analaysis

\begin{tabular}{|c|c|c|c|c|}
\hline & Normal control & FIP & FIP and saline & FIP and $80 \mu \mathrm{g} / \mathrm{kg}$ digoxin \\
\hline$M D A(n M / m g$ protein $)$ & $10.9 \pm 1.5$ & $54.4 \pm 6.1^{\star \star}$ & $43.8 \pm 2.2^{\star *}$ & $29.1 \pm 5.5^{\#}$ \\
\hline$I L-6(p g / m l)$ & $7.7 \pm 2.6$ & $25148 \pm 1021.5^{* *}$ & $22145.1 \pm 845.7^{\star *}$ & $11458.5 \pm 457.3^{\# \#}$ \\
\hline IL 1-Beta $(p g / m l)$ & $3.1 \pm 0.8$ & $2507.2 \pm 75.4^{* \star}$ & $2345.1 \pm 115.1^{\star *}$ & $689.6 \pm 45.8^{\# \#}$ \\
\hline TNF alfa $(p g / m l)$ & $14.5 \pm 2.3$ & $389.4 \pm 14.8^{\star *}$ & $415.2 \pm 13.4^{\star *}$ & $145.5 \pm 5.9^{\#}$ \\
\hline$C R P(m g / d l)$ & $0.52 \pm 0.1$ & $1.1 \pm 0.3^{*}$ & $1.2 \pm 0.1^{\star *}$ & $0.67 \pm 0.1^{\#}$ \\
\hline Lactic acid $(\mathrm{mmol} / \mathrm{L})$ & $1.4 \pm 0.2$ & $3.8 \pm 0.3^{*}$ & $3.7 \pm 0.1^{\star}$ & $2.2 \pm 0.2^{\#}$ \\
\hline
\end{tabular}

Note: ${ }^{*} p<0.05,{ }^{*} p<0.001$ different from normal groups; ${ }^{*} p<0.01,{ }^{\# *} p<0.001$ different from FIP and saline group.

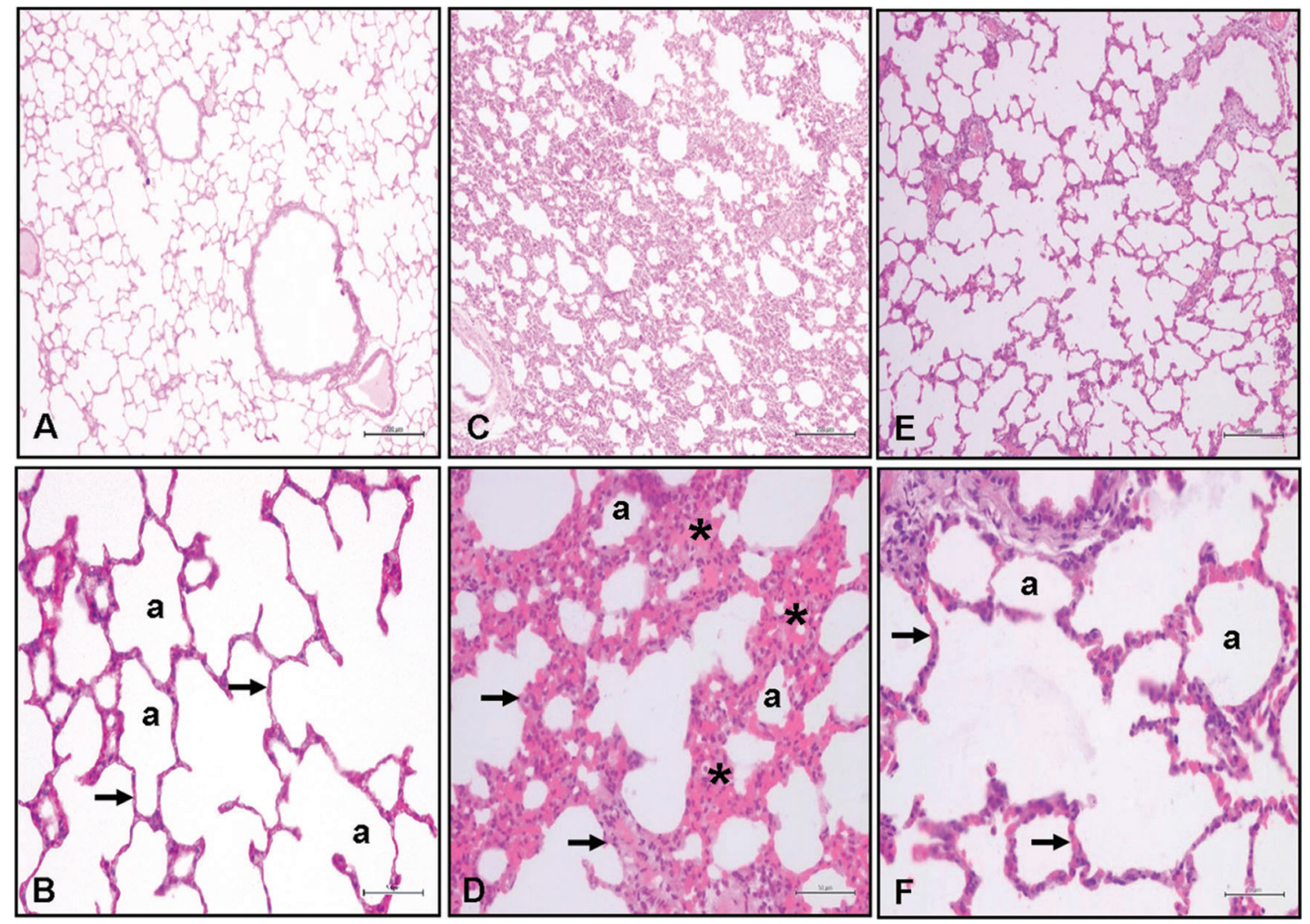

FIGURE 1. Histological sections of rat lungs. Lung histolopathology $\times 10$ and $\times 40$ magnification H\&E staining. A-B: Normal control group lung, (a) Alvelol, C-D: FIP and $10 \mathrm{ml} / \mathrm{kg} \% 0.9 \mathrm{NaCl}$ saline (plasebo) groups showed severe histopathologic alteration related to increased alveolar inflammation $\left(^{*}\right.$ ) and septal thickness (arrow), E-F: FIP and $80 \mu \mathrm{g} / \mathrm{kg}$ digoxin groups showed decreased inflammation and septal septal thickening (arrow).

TABLE 2

Histological evaluation of the lung

\begin{tabular}{lllll}
\hline & Normal control & FIP & FIP and saline & FIP and 80 $\boldsymbol{\mu g} / \mathbf{k g}$ digoxin \\
\hline$A C$ & $0.3 \pm 0.2$ & $3.4 \pm 0.4^{\star *}$ & $3.3 \pm 0.3^{*}$ & $1.7 \pm 0.3^{\#}$ \\
$H$ & $0.1 \pm 0.1$ & $1.7 \pm 0.2^{\star}$ & $1.6 \pm 0.1^{\star *}$ & $0.5 \pm 0.2^{\# \#}$ \\
$A L$ & $0.2 \pm 0.1$ & $2.4 \pm 0.3^{\star *}$ & $2.8 \pm 0.3^{\star *}$ & $1.3 \pm 0.3^{\#}$ \\
$P E$ & $0.3 \pm 0.2$ & $3.5 \pm 0.3^{\star *}$ & $3.1 \pm 0.3^{*}$ & $1.6 \pm 0.2^{\#}$ \\
$T A$ & $0.2 \pm 0.1$ & $3.1 \pm 0.2^{\star *}$ & $2.8 \pm 0.3^{\star *}$ & $1.4 \pm 0.2^{\# \#}$ \\
$C T$ Hounsfield unit $(\mathrm{HU})$ & $-659.5 \pm 13.4$ & $-521.2 \pm 10.1^{\star *}$ & $-545.8 \pm 17.3^{\star *}$ & $-638.5 \pm 6.7^{\# \#}$ \\
\hline
\end{tabular}



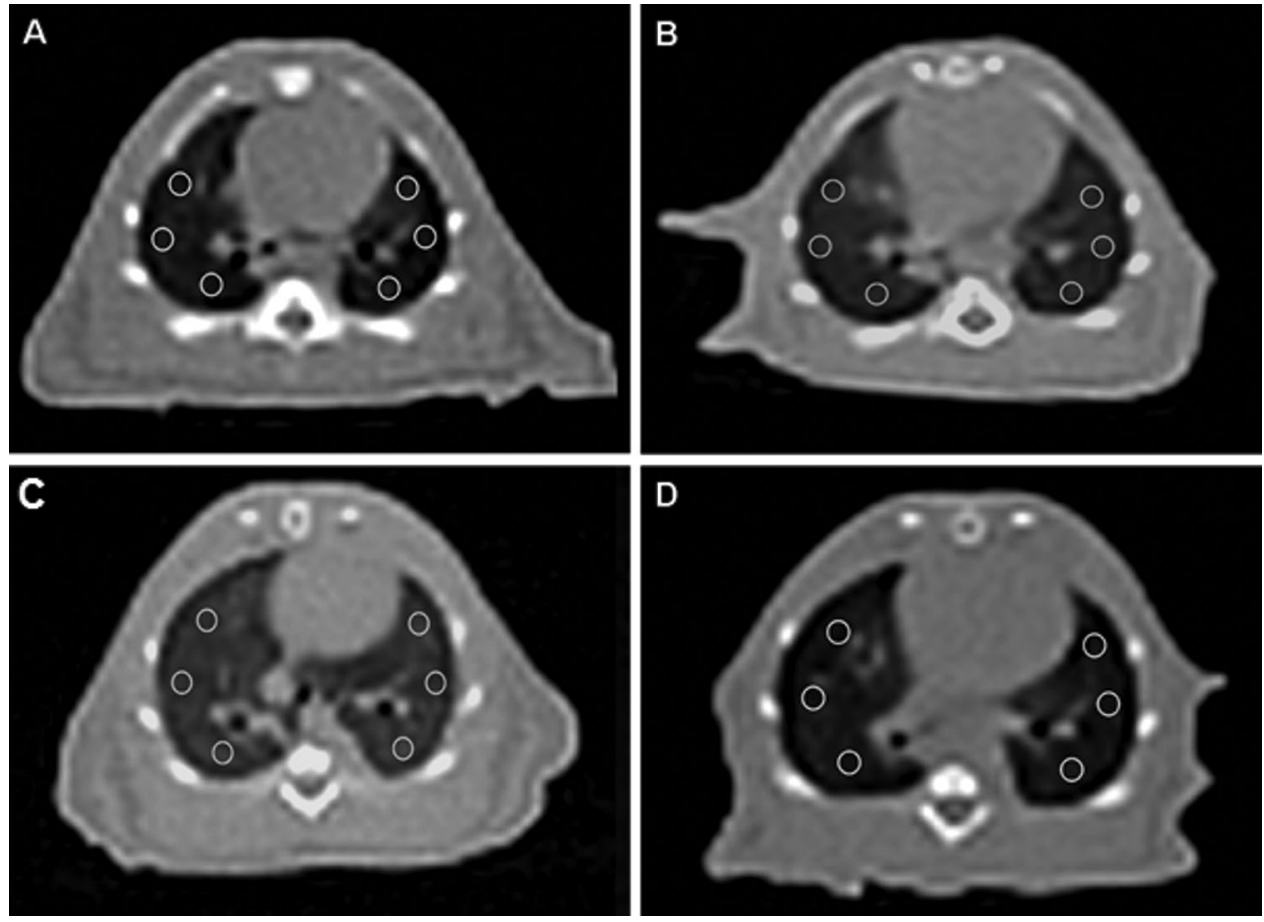

FIGURE 2. Computerized Tomography of Rats. Axial CT images of lung at the level of the heart, six ROI placed with the same size at the same location $A$ : Normal Control group lung, B: FIP group showed increased density of lung, C: FIP and $10 \mathrm{ml} / \mathrm{kg} \% 0.9$ $\mathrm{NaCl}$ saline (plasebo) group showed increased density of lung, D: FIP and $80 \mu \mathrm{g} / \mathrm{kg}$ digoxin group showed density of lung closer to normal group.

that the cardiac glycoside oubain reduced peritonitis findings and decreased inflammation to a negligible level. Furthermore, they found that cardiac glycosides reduced cytokine IL-1 $\beta$ and TNF-a levels, leukocyte infiltration, and edema formation, while they did not affect the viability or function of leukocytes (Leite et al., 2015). Taken together, these findings suggest that cardiac glycosides suppress acute inflammation.

MDA is a marker of oxidative stress. The decrease in the MDA value, which increased after the sepsis model, upon digitoxin administration indicated that the treatment reduced the oxygen requirement of the tissues by reducing both the cardiac and the systemic oxidative stress. Similar results were reported in a study in which digitoxin was used for cardiotonic purposes (Leite et al., 2015).

CRP is an indicator of the presence of the inflammatory process. The cardiac glycosides ouabain and digitoxin inhibit IL-1b- and IL-6-induced alpha-fetoprotein (AFP) expression (Peter et al., 2010). Inhibition of AFP synthesis also correlates with the potency of cardiac glycosides in $\mathrm{Na}^{+} / \mathrm{K}^{+}-$ ATPase. The inhibitory activity of cardiac glycosides on CRP expression may have important implications for the treatment of cardiovascular disease and other inflammatory processes (Peter et al., 2010). The decrease in CRP levels compared to the FIP and FIP+saline groups with digitoxin administration in our study is further evidence of antiinflammatory activity.

Lactate is one of the most important parameters both in the follow-up of patients with sepsis and in analyzing its severity. As soon as the oxygen demand of the tissues increases, the level of lactate, which is activated, rises (Svetlana et al., 2019). In our study, the lactate level increased in the FIP and FIP+ saline groups. The lactate level also improved significantly in the digitoxin treatment group compared to the other groups. This showed that the oxygen requirement of the tissues decreased after digitoxin treatment. The effect might have been due either to hemodynamic improvement secondary to the cardiac effect, or to a decrease in oxidative stress secondary to the antiinflammatory effect and a decrease in the energy requirement due to inhibition of the $\mathrm{Na}^{+} / \mathrm{K}^{+}$-ATPase pump.

In a study by Esposito, conducted to suppress the inflammatory response of pneumococcal pneumonia, high mortality was observed among the animals treated with digitoxin alone because the infectious agent was not eliminated (Esposito, 1985). By contrast, a high mortality rate was not observed in our study. This discrepancy might be due to the fact that we aimed to detect only the shortterm $(24 \mathrm{~h})$ effects of digitoxin. We have shown that the inflammatory process is suppressed by digitoxin and suggest that combination with appropriate antibiotic therapy could increase the effectiveness of the treatment.

ALI is one of the most common and fatal complications of sepsis (Filgueiras et al., 2012). The rapid prevention of acute complications of sepsis is made possible by inhibiting all the inflammatory processes. Our evaluation revealed that the use of digitoxin corrected impaired lung tissue histology (FIP and FIP+saline; severe histopathologic alteration related to increased alveolar inflammation $\left(^{*}\right)$ and septal thickness). (It reduced alveolar inflammation and septal thickening compared to FIP+saline). Bette et al. observed that digitoxin triggered an excessive immune response of proinflammatory cytokines in the host airways of pneumotropic viruses, including coronavirus disease 2019 (COVID-19), inhibited the formation of a cytokine storm, and $10 \mu \mathrm{g} / 100 \mathrm{~g}$ digitoxin administration led to similar improvements in the histological findings to those in our study in comparison to the normal group. In their study, the dose of digitoxin was given for 4 days. We observed similar results during the acute period after a single dose.

Our results by Gattinoni et al. (2001) in the evaluation made with the Hounsfield unit in the ROIs of Thorax CT, which was taken to confirm the findings, as in the 
radiological study (Gattinoni et al., 2001) performed by him, the aeration of the digitoxin group was found to be significantly higher than the FIP and FIP+saline groups. These results suggest that digitoxin ameliorates ALI by suppressing the excessive inflammatory response of sepsis during the early period.

\section{Limitations}

The duration of our study was limited to $24 \mathrm{~h}$, as we investigated the effects against sepsis after the first intervention; therefore, no exitus was observed between the groups. The longer-term effects of digitoxin on sepsis will be discussed in future studies. In addition, since we mainly investigated the anti-inflammatory effect of digitoxin in our study, there was no group that received antibiotic therapy. The clinical relevance of early treatment in sepsis is poor, except for early antibiotic therapy.

\section{Conclusion}

Digitoxin was observed to have curative effects in both radiological and histological evaluations of the lung, as well as biochemical healing in sepsis. We suggest that initiating the use of digitoxin in addition to the treatments recommended in the guidelines at the first presentation of sepsis will be protective against side effects such as ALI, and may reduce mortality and the need for mechanical ventilation by preventing a cytokine storm.

Availability of Data and Materials: The datasets used and/or analysed during the current study are available from the corresponding author on reasonable request.

Author Contribution: The authors confirm contribution to the paper as follows: study conception and design: Ejder Saylav Bora, Mümin Alper Erdoğan; data collection: Bahattin Özkul, İbrahim Halil Sever, İbrahim Söğüt; analysis and interpretation of results: Canan Hürdağ, Oytun Erbaş; draft manuscript preparation: Ejder Saylav Bora. All authors reviewed the results and approved the final version of the manuscript.

Ethics Approval: Animal Ethics Committee's consent (Science University, Ethical No. 02210623).

Funding Statement: The authors received no specific funding for this study.

Conflicts of Interest: The authors declare that they have no conflicts of interest to report regarding the present study.

\section{References}

Bette SP, Blancol JC, John R (2020). Pollard classical drug digitoxin inhibits influenza cytokine storm, with implications for Covid-19 Therapy. In Vivo 34: 3723-3730. DOI 10.21873/ invivo.12221.

Dec GW (2003). Digoxin remains useful in the management of chronic heart failure. Medical Clinics of North America 87: 317-337. DOI 10.1016/S0025-7125(02)00172-4.

Esposito AL (1985). Digoxin disrupts the inflammatory response in experimental pneumococcal pneumonia. Journal of Infectious Diseases 152: 14-23. DOI 10.1093/infdis/152.1.14.
Filgueiras LR Jr, Martins JO, Serezani CH, Capelozzi VL, Montes MB et al. (2012). Sepsis-induced acute lung injury (ALI) is milder in diabetic rats and correlates with impaired NFkB activation. PLoS One 7: e44987. DOI 10.1371/journal.pone.0044987.

Fürst R, Zündorf I, Dingermann T (2017). New knowledge about old drugs: The anti-inflammatory properties of cardiac glycosides. Planta Medica 83: 977-984. DOI 10.1055/s-0043-105390.

Gattinoni L, Caironi P, Pelosi P, Goodman LR (2001). What has computed tomography taught us about the acute respiratory distress syndrome? American Journal of Respiratory and Critical Care Medicine 164: 1701-1711. DOI 10.1164/ajrccm.164.9.2103121.

Hashim T, Elbaz S, Patel K, Morgan CJ, Fonarow GC et al. (2014). Digoxin and 30-day all-cause hospital admission in older patients with chronic diastolic heart failure. American Journal of Medicine 127: 132-139. DOI 10.1016/j.amjmed.2013.08.006.

Jagielska J, Salguero G, Schieffer B, Bavendiek U (2009). Digitoxin elicits anti-inflammatory and vasoprotective properties in endothelial cells: Therapeutic implications for the treatment of atherosclerosis? Atherosclerosis 206: 390-396. DOI 10.1016/j.atherosclerosis.2009.03.019.

Kwon WY, Suh GJ, Kim KS, Kwak YH (2011). Niacin attenuates lung inflammation and improves survival during sepsis by downregulating the nuclear factor- $\mathrm{kB}$ pathway. Critical Care Medicine 39: 328-334. DOI 10.1097/CCM.0b013e3181feeae4.

Leite JA, Alves AK, Galvão JG, Teixeira MP, Cavalcante-Silva LH et al. (2015). Ouabain modulates zymosan-induced peritonitis in mice. Mediators of Inflammation 2015: 1-12. DOI 10.1155/ 2015/265798.

Letícia V, Aline AS, Andreza MM, Flávio Martins de Oliveira, Débora Oliveira Lopes et al. (2018). 21-Benzylidene digoxin, a novel digoxin hemi-synthetic derivative, presents an anti-inflammatory activity through inhibition of edema, tumour necrosis factor alpha production, inducible nitric oxide synthase expression and leucocyte migration. International Immunopharmacology 65: 174-181. DOI 10.1016/j.intimp.2018.10.010.

Maurizio Z, Federica C, Luca F (2014). Overview of current lung imaging in acute respiratory distress syndrome. European Respiratory Review 23: 519-530. DOI 10.1183/09059180.00001314.

Nguyen HB, Rivers EP, Abrahamian FM, Moran GJ, Abraham E et al. (2006). Emergency Department Sepsis Education Program and Strategies to Improve Survival (ED-SEPSIS) Working Group (2006). Severe sepsis and septic shock: Review of the literature and emergency department management guidelines. Annals of Emergency Medicine 48: 28-54.e1. DOI 10.1016/j.annemergmed.2006.02.015.

Paul EM (2018). Vitamin C for the treatment of sepsis pharmacology therapeutics 2018. Pharmacology \& Therapeurtics 189: 6370. DOI 10.1016/j.pharmthera.2018.04.007.

Peter K, Andreas G, Schäfer S., Torzewski Jan (2010). Cardiac glycosides potently inhibit C-reactive protein synthesis in human hepatocytes. Biochemical and Biophysical Research Communications 394: 233-239. DOI 10.1016/j.bbrc.2010.02.177.

Remick DG, Bolgos G, Copeland S, Siddiqui J (2005). Role of interleukin-6 in mortality from and physiologic response to sepsis. Infection and Immunity 73: 2751-2757. DOI 10.1128/IAI.73.5.2751-2757.2005.

Shrum B, Anantha RV, Xu SX, Donnelly M, Haeryfar SM et al. (2014). A robust scoring system to evaluate sepsis severity in an animal model. BMC Research Notes 7: e28. DOI $10.1186 / 1756-0500-7-233$.

Svetlana H, Courtney EB, Alex RS, Yosuf WS, Ognjen Gajic et al. (2019). Hemodynamic profiles following digoxin use in 
patients with sepsis in the ICU. Journal of Critical Care 54: 175-179. DOI 10.1016/j.jcrc.2019.08.026.

Teijaro JR, Walsh KB, Long JP, Tordoff KP, Stark GV et al. (2014). Protection of ferrets from pulmonary injury due to H1N1 2009 influenza virus infection: Immunopathology tractable by sphingosine-1- phosphate 1 receptor agonist therapy. Virology 452-453: 152-157. DOI 10.1016/j.virol.2014.01.003.

Tyml K, Swarbreck S, Pape C, Secor D, Koropatnick J et al. (2017). Voluntary running exercise protects against sepsis-induced early inflammatory and pro-coagulant responses in aged mice. Critical Care 21: 81. DOI 10.1186/s13054-017-1783-1.

Vincent JL, Pereira AJ, Gleeson J, Backer D (2014). Early management of sepsis. Clinical and Experimental Emergency Medecine 1: 3-7. DOI 10.15441/ceem.14.005.

Yang M, Cao L, Xie M, Yu Y, Kang R et al. (2013). Chloroquine inhibits HMGB1 inflammatory signaling and protects mice from lethal sepsis. Biochemical Pharmacology 86: 410-418. DOI 10.1016/j.bcp.2013.05.013. 